\title{
Estudio de la ansiedad producida por la actuación entre los estudiantes de música noruegos
}

\author{
Marianne Kaspersen \\ K. Gunnar Götestam
}

Departamento de Psiquiatría y Medicina

Comportamental, Universidad de Ciencia

y Tecnología de Noruega, Thondheim

NORUEGA

\begin{abstract}
RESUMEN - La realización del presente estudio está basada en una encuesta sobre la existencia de ansiedad durante la actuación musical (AAM) de los estudiantes noruegos de conservatorio de música,analizando las diferencias respecto a sexo,nivel de estudios,instrumentos y tipo de programa educativo. Un total de 126 participantes (índice de respuesta del 96,2\%) completó el cuestionario,que contenía preguntas sobre fundamentos musicales, percepción de la ansiedad durante la interpretación y un listado de síntomas y de ciertas variables relacionadas con la AAM. Además se incluyó una pregunta acerca de si los encuestados necesitaban ayuda en relación a los problemas de la AAM. Los resultados revelan que la AAM supone un problema entre los estudiantes de música noruegos. En la muestra total, un $36,5 \%$ de los participantes necesitó de ayuda en problemas relacionados con la AAM.

Además, se observó que la ansiedad, tanto antes como durante la interpretación,está asociada con una afectividad negativa y no con la afectividad positiva. Además existían diferencias entre los sexos con respecto a la ansiedad percibida, los síntomas de la AAM y la "necesidad de ayuda". En cambio hubo diferencias importantes entre los dos niveles educativos. Los pianistas y los músicos de cuerda manifestaron que con frecuencia sufrían de una "alta ansiedad". Se detectó una importante y drástica diferencia en los síntomas entre los estudiantes de la "línea de jazz", con un nivel de ansiedad sustancialmente inferior, frente a los alumnos de música clásica. Los resultados obtenidos ofrecen consecuencias pedagógicas para los profesores en los niveles más avanzados de la educación musical.
\end{abstract}

\section{Introducción}

La ansiedad durante la actuación de los músicos y estudiantes de música no ha sido objeto de grandes investigaciones, no obs- tante, existen muchos estudios que han confirmado que el problema de este tipo de ansiedad (ansiedad durante la actuación musical: AAM) existe entre ciertos grupos de músicos, como son los de orquesta (Step- 
toe \& Fidler 1987, Fishbein y col. 1988) y los estudiantes de música (Abel \& Larkin 1990, Wesner y col. 1990, Kivimäki 1995). Concretamente Wesner y col. (1990) observaron que un $16,5 \%$ de una población estudiantil compuesta por 302 encuestados se encontraba muy ansiosa durante las actuaciones y que un $9,0 \%$ evitaba con cierta frecuencia determinadas interpretaciones debido a la ansiedad. Además, Clark y Agras (1991) vieron que el 97\% de los 94 músicos con AAM realizaban actuaciones defectuosas a causa de la ansiedad, mientras que un $77 \%$ del mismo grupo las evitaba por ese mismo motivo.

Fishbein y col. (1987) vieron que el miedo escénico era un problema en un $24 \%$ del total de 2.212 músicos de orquesta en los Estados Unidos. Por su parte Wesner y col. (1990) se dieron cuenta de que un $41,1 \%$ de los estudiantes y de los miembros de la Escuela de Música de la Universidad de Iowa estaban entre moderada y altamente trastornados por síntomas relacionados con la AAM. Además de esto, Clark (1989) calificó la AAM como el problema psicológico más común entre músicos. Por si fuera poco, una encuesta nacional realizada entre los músicos de orquesta en los Estados Unidos dio como resultado que era el miedo escénico el problema denunciado con mayor frecuencia (junto con el esfuerzo ocular) y el de mayor gravedad (Fishbein y col. 1988).

Se han empleado muchos términos para caracterizar los problemas relacionados tanto con la interpretación como con las situaciones propias de la actuación. El miedo escénico, la ansiedad durante la actuación y la ansiedad durante la interpretación han sido utilizados de forma intercambiable para designar a cierta clase de ansiedad relacionada con la interpretación (Brodsky 1997). No obstante, los términos no son iguales. En este artículo, el término AAM se utilizará para designar a la ansiedad subjetiva antes y durante la interpretación ante la audiencia, entre los alumnos de música del conservatorio que estudiaban a tiempo completo interpretación musical, sin estar incluidos músicos profesionales en ejercicio.

Sin embargo, los participantes son aspirantes a convertirse en músicos profesionales y, como tales, son vistos como músicos y no como aficionados puesto que todos ellos han pasado por un periodo de tres días de exámenes de disciplinas tanto teóricas como instrumentales. En Noruega este tipo de exámenes son los mismos para todas las escuelas superiores de educación musical. Por todo ello se trata de músicos, pero no músicos profesionales sino estudiantes, y por lo tanto estamos ante un grupo bastante homogéneo.

La ansiedad durante la actuación y la calidad de la misma cumplen con la ley YerkesDodsons (Steptoe \& Fidler 1987, Salmon 1990). Por ello, si la AAM sigue esta ley, una moderada cantidad de ansiedad debe dar como resultado unas condiciones óptimas en la actuación. Esto, de todas formas, es difícil de determinar por dos razones. Primero, porque los síntomas fisiológicos y cognitivos no están, necesariamente sincronizados (Salmon 1990). La ansiedad cognitiva, por ejemplo, muestra de forma probada un acostumbramiento más lento que la propia ansiedad fisiológica (Salmon 1990). Segundo, porque lo que se considera ansiedad moderada depende de la manera en que cada individuo la interprete. Además, según Wilson (1994), la forma de U invertida califica a la AAM de una forma aún más complicada. Según Wilson (1994), los rasgos de ansiedad, la destreza en la tarea y el grado de stress en una situación determinada deben ser tomados en consideración. Esto implica, según Hargrea- 
ves \& North (2000), que los individuos con altos rasgos de ansiedad actuaran mejor cuando perciben su tarea como algo sencillo al tiempo que la presión de la situación es percibida como baja, mientras que aquellos otros individuos que poseen niveles inferiores de ansiedad, actuarán mejor cuando la dificultad sea lo suficientemente alta y las condiciones de actuación supongan un mayor desafío.

Se han estudiado además las relaciones existentes entre la AAM y la fobia social (Clark \& Agras 1991), entre la AAM y la sensibilidad a la ansiedad (Barney Dews \& Williams 1989, Salmon 1990) y entre la AAM y el neuroticismo (Steptoe \& Fidler 1987). Los músicos socialmente más ansiosos parecen más tender a experimentar peores consecuencias en relación con los síntomas de la AAM. También existen otro tipo de factores implicados: factores de personalidad tales como la ansiedad-rasgo y la ansiedadestado (Kemp 2000), así como la autoestima (Barney Dews \& Williams 1989). Se han considerado como importantes factores sociales algunos como "el perfeccionismo socialmente prescrito" (Mor y col. 1995). Además se han sugerido aspectos motivacionales (Barney Dew \& Williams 1989). Por todo ello, se debería esperar que la AAM se modificara en relación con las diferentes variables de la personalidad y dentro de ellas, según el nivel del neuroticismo y los rasgos de la ansiedad.

Probablemente por esto, la AAM esté relacionada con muchas variables fisiológicas y sociales, y haya sido considerada, según algunos autores, como formada por diferentes componentes (Salmon 1990, Craske \& Craig 1984, Lehrer 1987). Uno de ellos consistiría en respuestas de alerta fisiológica o autonómica (como por ejemplo el latido del corazón, los temblores y la sudoración), otro componente sería cogniti- vo (como la autoafirmación y las autoinstrucciones previas, durante y después de la interpretación) y otro componente sería comportamental palpable (como los problemas técnicos con el instrumento, los problemas de interacción con los maestros y el hecho de evitar hacer cosas que incrementen la ansiedad) (Lehrer 1987). De esta forma, se puede entender que la AAM está formada por la reacción fisiológica de alerta y la ansiedad cognitiva compuesta por pensamientos acerca de las evaluaciones que hace la audiencia, así como de las evaluaciones continuas de sus propias habilidades.

Se ha demostrado que la AAM varía según las condiciones de interpretación. Las actuaciones en solitario parecen producir mayor ansiedad que las de orquesta o práctica (Hargreaves \& North 2000). A tenor de ello, el nivel de preparación es importante, especialmente cuando se está actuando de memoria (Salmon 1990). Se ha visto también que hay aspectos sociales de importancia tales como la cantidad de público (Jackson \& Latané 1981) o el tipo de audiencia.

Basados en lo anterior, esperamos encontrar diferencias entre aquellos músicos que tocan instrumentos en la orquesta y aquellos otros que tocan como solistas. También creemos que la AAM esté relacionada con las condiciones de la audiencia y el nivel de preparación.

Ciertos descubrimientos apuntan al hecho de que podría haber diferencias entre los sexos en lo que se refiere a la ansiedad durante la actuación (Kubansky \& Stewart 1999). Craske y Craig (1984) observaron que las mujeres muestran mayores niveles de ansiedad que los hombres tras la aplicación de un cuestionario. Además, las mujeres exhiben una menor reacción de alerta 
autónoma cuando se las compara con hombres (Craig 1968). Craske y Craig (1984) vieron además como ciertos patrones de respuesta entre pianistas hombres y pianistas mujeres eran diferentes y ello se daba en lo que se refiere al estado fisiológico de alerta y a las autoinstrucciones cognitivas. Entre las mujeres era más probable recoger manifestaciones personales negativas justo antes de la actuación, mientras que los hombres revelaban un mayor estado autonómico de alerta en relación con las actuaciones. Además,Abel y Larkin (1990) observaron cómo las mujeres mostraban una mayor ansiedad subjetiva que los hombres, mientras que estos exhibían una presión sanguínea sistólica mayor al compararlos con las mujeres. Resultados similares encontraron Craske y Craig (1984). Nosotros esperamos ver diferencias de la AAM según el sexo de los músicos.

Si los sistemas de respuesta están desincronizados, uno debe esperar la combinación de baja ansiedad fisiológica y alta ansiedad cognitiva, dando como resultado reacciones de evitación, por el hecho de que las ansiedades cognitivas pueden aumentar el miedo ante la posibilidad de experimentar síntomas de ansiedad fisiológica. Esta combinación también puede ceder ante determinados síntomas intrusivos como la meditación acerca de las situaciones vividas durante la actuación, lo cual se parece a la ansiedad expectante y a la ansiedad previa a la interpretación.

El conocimiento de dichas diferencias de grupo pueden interesar a los profesores de música. Una detección de aquellas características que diferencian a los estudiantes más vulnerables a la AAM, de los que no lo sean, es importante para los profesores de todos los niveles de la enseñanza musical, aunque esta investigación se refiere más a alumnos de Conservatorio de Música que aspiran a convertirse en músicos profesionales.

Comparada con el resto de los países europeos, Noruega no tiene tradicionalmente altos estándares de actuación entre los estudiantes de música. Hasta hace poco, los músicos de mayor talento tenían que irse a otros países de Europa para poder seguir allí con su carrera profesional (Herresthal 1993). Incluso hoy es todavía corriente que los estudiantes noruegos vayan a otros países como el Reino Unido, Alemania, Italia, Francia o los Estados Unidos a estudiar. No ha sido, hasta hace poco, que Noruega ha tenido músicos internacionalmente conocidos sin necesidad de haberse formado en el extranjero. Por lo tanto nos parece razonable concluir que Noruega es aún más inmadura en lo que respecta a determinadas facetas de la cultura musical, que el resto de Europa (Norbert 1994). Ello podría estar relacionado con el proceso cultural del último siglo,que puede haber retrasado algunos aspectos internacionales de la cultura noruega. En Noruega, por lo tanto, existe una "frescura" o un cierto "carácter reciente" en lo referente a su cultura musical,por tanto el estudio de una muestra noruega permite evaluar el desarrollo de la AAM, así como en qué orden afecta a grupos diferentes en sus fases más tempranas, al tiempo que ofrece la posibilidad de comparar sus resultados con lo que sucede en otros países europeos.

El propósito de este estudio es triple: primero, valorar la frecuencia y los problemas relacionados con la AAM, así como la necesidad de ayuda que precisan los estudiantes noruegos del conservatorio de música; segundo, investigar las características de la AAM y la necesidad de ayuda en relación con el sexo, el nivel de estudios, el tipo de instrumentos y el programa de estudios; y tercero, examinar los síntomas asociados 
con la ansiedad y con ello ver si estas diferencias están relacionadas con la naturaleza de la afectividad negativa (NA) y la afectividad positiva (PA).

\section{Métodos}

\section{Individuos}

Se pidió la participación de alumnos del Conservatorio de Música a tiempo completo, que estaban estudiando en ese momento, como graduados o aspirantes, en dos conservatorios de Noruega. Las dos escuelas que participaron en este estudio constituyen una selección representativa de la población total de los estudiantes de música de Noruega (aproximadamente un 20 por ciento de la población total de los estudiantes de música en este país). La muestra estaba formada por $51(40,5 \%)$ hombres y $75(59,5 \%)$ mujeres con un rango de edad entre los 19 y los 30 años (una media de 22,0). De ellos setenta y siete $(63,6 \%)$ alumnos eran aspirantes a graduado, cuarenta y cuatro $(36,4 \%)$ eran graduados y cinco de ellos no indicaron su nivel. Todos los estudiantes participaban en la orquesta o actuaban como solistas poseyendo una amplia experiencia en las actuaciones.

En uno de los dos conservatorios de música existía un desequilibrio en la distribución de los estudiantes de jazz (19) y de "música clásica” (107), por lo que se realizaron sólo comparaciones cualitativas, sugiriendo algunas consideraciones para posteriores investigaciones. Sin embargo, se hizo análisis de diferencias con respecto a los tipos de instrumentos, al ser estos grupos más nivelados en su tamaño.

Estaban representados los nueve tipos de instrumentos, 25 erán músicos de teclado,
21 vocalistas, 10 de metal, 27 músicos de cuerda, 5 flautistas, 3 percusionistas, 5 guitarristas y 6 músicos de instrumentos de viento (de madera). Además 17 de los 19 estudiantes de jazz indicaron el instrumento musical que manejaban (saxofón, guitarra, bajo, piano, trompeta, percusión y vocal). Siete del total no indicaron el instrumento musical.

\section{Procedimiento}

Los participantes fueron informados acerca de esta investigación mediante posters e información dada por la administración, comunicándoles que la participación era voluntaria. Se procedió a la entrega de las preguntas durante una hora de descanso y en un día en el que se pudiera reunir a tantos estudiantes como fuera posible. Del total de 131 cuestionarios entregados, 126 fueron devueltos $(96,2 \%)$.

\section{Cuestionario}

Se elaboró un cuestionario basado en una revisión bibliográfica sobre la AAM y en el conocimiento general acerca de la tradición del Conservatorio de Música de Noruega. Dicho cuestionario está formado por aspectos relativos a variables demográficas, fundamentos musicales, experiencia en actuaciones, una sección sobre la ansiedad percibida en la actuación y otra sección sobre los diferentes factores sociales, situacionales y físicos, relacionados con la AAM (variables dependientes de la ansiedad). Además se incluyó una pregunta abierta sobre técnicas para abordar estos problemas y otra serie de preguntas dirigidas a evaluar la necesidad de ayuda, así como la presencia de otro tipo de ansiedades. El cuestionario anima- 
ba a los participantes a hacer comentarios más profundos sobre sus experiencias en la interpretación, lo cual fue proporcionado por el $86,5 \%$ de los estudiados. Al final del cuestionario se incluía la Escala de Afectos Positivos y Negativos, PANAS (Watson y col. 1988). Este cuestionario presenta medidas sobre la afectividad positiva (PA) o la extroversión y la afectividad negativa (NA), lo cual es también una medida de rasgos de la afectividad (Lonigan y col. 1999) y de neuroticismo (Eysenck y col. 1985).

\section{Preliminares}

El análisis de la fiabilidad de todos los síntomas (excepto el ítem número ocho, que falló a la hora de ajustarse al criterio de $\phi>.3$ posee un Crohnbachs $\alpha=.73$. El análisis factorial con rotación Varimax ofreció dos grupos de síntomas. Uno de los grupos estaba relacionado de forma clara con la reacción de alerta, Crohnbachs $\alpha=.58$ consistente en tres ítems. El otro grupo incluía elementos cognitivos sobre evaluaciones sociales y de la situación general de la actuación, y dio un resultado de Crohnbachs $\alpha=.67$. Este estaba compuesto de cinco ítems. Las "Variables dependientes de la Ansiedad" no dieron, mediante métodos de reducción de datos, ningún factor consistente y por lo tanto fueron tratados como ítems en los análisis. El análisis de la fiabilidad dio un resultado de Crohnberg $\alpha$ $=.72$. Estos ítems se tuvieron en cuenta como medida general de presión en una situación de actuación musical. La lista de síntomas variables se trató como ítems, además de ser considerados como clusters o grupos.

En el análisis final, aquellos que informaron una ansiedad variable fueron exclui- dos del análisis y tratados como datos perdidos. Esto se hizo con el fin de poder tratar la ansiedad variable como variable continua.

\section{Resultados}

En la muestra total, el 5,6\% decía no experimentar ansiedad antes de las actuaciones. Un 74,7\% informó acerca de un grado específico de ansiedad y el 16,7\% decía tener ansiedad variable antes de la actuación. En cuanto a la ansiedad durante la actuación, el 7,9\% no la experimentó frente al $70,6 \%$ que dijo sentir cierto grado específico de ansiedad, y un 19,8\% decía experimentar una ansiedad variable (tabla I). La evitación debida a la AAM fue detectada en el 5,6\% mientras que el $12,7 \%$ decía tener pensamientos intrusivos sobre las actuaciones en la vida ordinaria. Además, el $36,5 \%$ expresó la necesidad de ayuda para superar la ansiedad de la actuación, porcentaje bastante diferente para ambos sexos (ver tabla I).

El 39,7\% presentó ansiedad en otras situaciones distintas de las relacionadas con la actuación, las cuales en el 34,9\% parecían estar relacionadas con situaciones de índole social. Además, aquellos alumnos que se identificaban como músicos, parecían tener menor necesidad de ayuda entre los que dijeron sentirse inseguros a la hora de identificarse como músicos, 31 (51,6\%) precisaban ayuda, mientras que sólo 13 $(42,18 \%)$ de aquellos que poseían una identidad musical bien establecida, indicaron tal necesidad. Un total de $61(48,4 \%)$ de los participantes utilizaban técnicas de ayuda para enfrentarse a la AAM, siendo hasta un total de diecisiete tipos de técnicas las utilizadas en estos casos; la mayoría utilizaba 
Tabla I

Porcentajes de necesidad de ayuda, AAM, pensamientos intusivos y evitaciones según sexo $(\mathrm{N}=126)$

\begin{tabular}{lccc} 
Variables & Total $(\mathrm{n}=126)$ & Hombres $(\mathrm{n}=51)$ & Mujeres $(\mathrm{n}=75)$ \\
\hline Necesidad de ayuda & 36,5 & 11,8 & 53,3 \\
Ansiedad antes de la interpretación & & & \\
$\quad$ Calma total & 5,6 & 3,9 & 6,7 \\
Un poco ansioso & 42,9 & 60,8 & 30,7 \\
Ansiedad pero que no requiere mucha concentración & 15,1 & 19,6 & 12,0 \\
Muy ansioso que precisa mucha concentración & 40 & 0,0 & 6,7 \\
Tal ansiedad que pienso que no irá bien & 12,7 & 7,8 & 16,0 \\
La ansiedad varía mucho & 16,7 & 7,8 & 22,7 \\
Ansiedad durante la interpretación & & & \\
Calma total & 7,9 & 13,7 & 4,0 \\
Un poco ansioso & 46,8 & 49,0 & 45,3 \\
Ansiedad pero que no requiere mucha concentración & 9,5 & 15,7 & 5,3 \\
Muy ansioso que precisa mucha concentración & 4,8 & 0,0 & 8,0 \\
Tal ansiedad que pienso que no irá bien & 9,5 & 9,8 & 9,3 \\
La ansiedad varía mucho & 19,8 & 11,8 & 25,3 \\
Pensamiento intrusivo sobre la actuación en la vida diaria & 12,7 & 7,8 & 16,0 \\
Evitar actuar & 5,6 & 5,9 & 5,3 \\
\hline
\end{tabular}

técnicas de meditación, de pensamientos positivos, de visualizaciones, o de respiraciones. Un total de $43(32,3 \%)$ mujeres y 18 $(9,2 \%)$ hombres empleaba al menos una técnica de ayuda.

Se pidió a los alumnos que dieran la razón de por qué estudiaban música y de ellos el 57,9\% lo hacían porque "amaban la música”. Por variadas necesidades musicales lo hacían el $17,5 \%$ de los estudiantes, mientras que un $8,7 \%$ lo hacía por "circunstancias accidentales".

En la tabla II se presentan los síntomas que padecían. Los temblores son los sínto-

Tabla II

Síntomas según sexo $(\mathrm{N}=126)$

Síntomas

Hombres $(\mathrm{n}=51)$ Mujeres $(\mathrm{n}=75) \quad$ Valor de $\mathrm{P}$

\begin{tabular}{|c|c|c|c|}
\hline Palpitaciones $^{1}$ & $20(39,2 \%)$ & $49(65,3 \%)$ & $\mathrm{p}<, 01$ \\
\hline Sudoración & $21(41,2 \%)$ & $32(42,7 \%)$ & n.s. \\
\hline Temblores & $24(47,1 \%)$ & $44(58,7 \%)$ & n.s. \\
\hline Sentimiento de pánico & $2(3,9 \%)$ & $10(13,3 \%)$ & n.s. \\
\hline Ideas de que no saldrá bien & $8(15,7 \%)$ & $32(42,7 \%)$ & $\mathrm{p}<, 01$ \\
\hline Sentimiento de disconfort ante la presencia de la gente & $3(5,9 \%)$ & $9(12,0 \%)$ & n.s. \\
\hline $\begin{array}{l}\text { Ideas de que el público piensa que no lo estoy haciendo } \\
\text { lo suficientemente bien }\end{array}$ & & & $n<05$ \\
\hline Miedo a que la gente me vea nervioso & $\begin{array}{r}5(9,8 \%) \\
5(3,5 \%)\end{array}$ & $\begin{array}{r}45(3,5 \%) \\
9(12,0 \%)\end{array}$ & $\begin{array}{c}\mathrm{p}<, 0 \mathrm{~s} \\
\text { n.s. }\end{array}$ \\
\hline Miedo a cometer algún error que pueda ver o escuchar el público & $11(21,6 \%)$ & $26(34,7 \%)$ & n.s. \\
\hline
\end{tabular}

${ }^{1}{ }^{1}$ El test $\chi^{2}$ se utiliza para establecer resultados significativos. 
mas más nombrados, aunque no existen resultados estadísticamente significativos según el sexo de pertenencia. Sin embargo tres síntomas sí que aparecieron como estadísticamente significativos según el sexo de estos músicos. Así sucedió con las "Palpitaciones", $\left(\chi^{2}=8,36, \mathrm{df}=1, \mathrm{p}<0,01\right)$, "Pensar que no me va a salir bien", $\left(\chi^{2}=10,20\right.$, df $=1, \mathrm{p}<0,01$ ), y "Pensar que la audiencia no cree que yo toque lo suficientemente bien" $\left(\chi^{2}=4,90, \mathrm{df}=1, \mathrm{p}<, 05\right)$. El resto de los síntomas no fueron estadísticamente significativos con respecto al sexo, no obstante lo cual las mujeres mostraron una tendencia a puntuar más alto en cada síntoma. Ver tabla II.

Cuatro de las variables de la "ansiedad dependiente" eran estadísticamente significativas con respecto al sexo. Las opciones: "La ansiedad depende de si estoy actuando en solitario o en grupo" $\left(\chi^{2}=3,72, \mathrm{df}=1, \mathrm{p}\right.$ $<0,05)$, "La ansiedad depende de cómo esté de cansado" ( $\chi^{2}=3,51$, df $=1, \mathrm{p}<$ $0,05)$, "La ansiedad depende de cómo me sienta de estresado" $\left(\chi^{2}=6,47, \mathrm{df}=1, \mathrm{p}<\right.$ $0,01)$, y "La ansiedad depende de la importancia que le doy a que todo esté perfectamente interpretado" $\left(\chi^{2}=6,01, \mathrm{df}=1, \mathrm{p}<\right.$ $0,05)$, fueron marcados por las mujeres con más frecuencia.

Los análisis de correlación entre sexo y nivel de la educación musical por un lado, y ansiedad y "necesidad de ayuda" por el otro, están representados en la tabla III (arriba).

Cuando se analizó la ansiedad atendiendo el nivel educativo musical, la palpitación era el único síntoma que resultó estadísticamente significativo $\left(\chi^{2}=11,05, \mathrm{df}=1, \mathrm{p}<0,01\right)$.

Tabla III

Correlaciones según sexo y nivel educativo (arriba) y correlaciones entre afectividad negativa, positiva, grupos de ansiedad y variables de ansiedad (abajo) $(\mathrm{N}=126)$

\begin{tabular}{lcc} 
Variable & Sexo & Nivel educativo \\
\hline Ansiedad antes de la actuación $^{1}$ &, $21^{*}$ &, 03 n.s. \\
Ansiedad durante la actuación $^{1}$ &, $12 \mathrm{n} . \mathrm{s}$. &, $08 \mathrm{n} . \mathrm{s}$. \\
Necesidad de ayuda $^{2}$ & $18,9(\mathrm{df}=1)^{* * *}$ &, $06(\mathrm{df}=1)$ n.s. \\
Variable & Afectividad negativa & Afectividad positiva \\
\hline Arousal &, $32^{* *}$ &, 61 \\
Cognitiva &, $37^{* *}$ &,$- 23 *$ \\
Ansiedad antes de la actuación &, $35^{* *}$ &,- 18 \\
Ansiedad durante la actuación &, $32^{* *}$ &,- 15
\end{tabular}

\footnotetext{
$\left({ }^{1}\right)=r_{p b}$ correlaciones.

$\left({ }^{2}\right)=\chi^{2}$ test.

(*) $\mathrm{p}<, 05$.

$(* *) \mathrm{p}<, 01$.
} 
Cincuenta y dos estudiantes $(67,5 \%)$ con un nivel inferior a la graduación, informaron sobre este síntoma, frente a $16(36,4 \%)$ de los graduados. También se manifestó con más frecuencia entre los alumnos graduados la evitación, de modo que en un $13,4 \%$ de los de las clases de graduados lo hizo, comparado con un $2,6 \%$ de los niveles inferiores $\left(\chi^{2}=4,07, \mathrm{df}=1, \mathrm{p}<0,5\right)$.

El ANOVA fue estadísticamente significativo con respecto a la ansiedad antes de una actuación $(\mathrm{F}=4,99 ; \mathrm{df}=8 ; \mathrm{p}<, 000)$ y durante la actuación entre los distintos grupos instrumentales $(\mathrm{F}=3,74$; $\mathrm{df}=8$; $\mathrm{p}<$ ,001).

El test Sheffé de diferencias de grupo mostró que eran estadísticamente significativas durante las actuaciones en el caso de los músicos de instrumentos de teclado y los músicos de jazz ( $\mathrm{md}=1,44 ;<, 05)$ y antes de la actuación entre los músicos de cuerda y jazz ( $\mathrm{md}=1,56 ; \mathrm{p}<, 05)$. No hubo diferencias entre los grupos instrumentales en cuanto a la "necesidad de ayuda". Las correlaciones existentes entre variables dependientes de la ansiedad, y la ansiedad, se representan en la tabla III (abajo).

Los resultados entre los estudiantes de música clásica y los de jazz no fueron analizados estadísticamente, sin embargo, se dio también una diferencia bastante drástica en cuanto a los síntomas existentes entre los alumnos de jazz, que presentaron síntomas de ansiedad menores, comparados con los estudiantes de música clásica. También resultó que $3(15,8 \%)$ de los que estudiaban el programa de jazz informaron acerca de la necesidad de ayuda, mientras que un total de $38(43,2 \%)$ del programa de música clásica, hicieron lo mismo.

Una inspección más minuciosa de los datos cualitativos (comentarios sobre experiencias personales), reveló diferentes actitudes entre los estudiantes de jazz y los de música clásica con respecto a las actuaciones. Diez de los diecinueve estudiantes de jazz comentaron que tocar frente a una audiencia les estimulaba. Estos mostraron las siguientes respuestas: "tocar frente a una audiencia me produce placer y un sentimiento excitante" o "tocar frente a una audiencia es pura adrenalina". Hubo cinco estudiantes que no contestaron a esta pregunta, y cuatro dijeron que "tocar frente a una audiencia puede ser una experiencia fantástica o terrible". A diferencia de estos los estudiantes de música clásica presentaban sentimientos desagradables ("destroza mi auto-confianza", "me pone tenso", "me hace sentir mucha presión", "presiento que esperaban más de mí", "me avergüenza", "es humillante", "es frustrante", "me dan náuseas" o "me molesta"). Sin embargo, estos resultados han sido analizados estadísticamente, y sólo muestran una pequeña diferencia entre los estudiantes de jazz y los estudiantes de música clásica con respecto a actitudes y sentimientos durante las actuaciones musicales, por lo que es preciso una investigación más profunda para analizar tales diferencias.

En este estudio, no se relacionaron las variables referentes a ansiedad y a necesidad de ayuda, con respecto a la ansiedad social. La ansiedad previa a la actuación solía estar asociada positi vamente a la afectividad negativa (NA) $(\mathrm{r}=, 35 ; \mathrm{p}<, 01)$ así como a la ansiedad durante la interpretación $(\mathrm{r}=, 32 ; \mathrm{p}<, 05)$. En cuanto a la necesidad de ayuda se asoció también con la NA $\left(\mathrm{r}_{\mathrm{pb}}=\right.$ ,23; $\mathrm{p}<, 05)$. Además, la ansiedad de antes y de después de la actuación estaba relacionada con muchas de las "variables dependientes de la ansiedad". (Ver tabla IV para más detalles). 
Tabla IV

Síntomas según el tipo de programa

\begin{tabular}{lcr} 
Síntomas & Clásica $(\mathrm{n}=107)$ & Jazz $(\mathrm{n}=19)$ \\
\hline Palpitación & $55(62,5 \%)$ & $6(31,6 \%)$ \\
Sudoración & $44(50,0 \%)$ & $5(26,3 \%)$ \\
Temblor & $55(62,5 \%)$ & $5(26,3 \%)$ \\
Sentimiento de pánico & $12(13,6 \%)$ & $0(0,00 \%)$ \\
Ideas de que no saldrá bien & $33(37,5 \%)$ & $3(15,8 \%)$ \\
Sensación de disconfort ante la presencia de gente & $11(12,5 \%)$ & $1(5,3 \%)$ \\
Ideas de que el público piensa que no lo estoy haciendo & & \\
$\quad$ lo suficientemente bien & $41(46,6 \%)$ & $12(63,2 \%)$ \\
Miedo a que la gente me vea nervioso & $9(10,2 \%)$ & $1(5,3 \%)$ \\
Miedo a cometer algún error que pueda ver o escuchar el público & $26(29,5 \%)$ & $5(26,3 \%)$ \\
\hline
\end{tabular}

\section{Discusión}

Los resultados indican que la AAM supone un problema entre los estudiantes de música noruegos. En la muestra total, el $36,5 \%$ informó sobre la necesidad de ayuda en problemas relacionados con la AAM. Además, el 16,7\% dijo estar, "muy ansioso" o "tan ansioso que creo que no saldrá bien" antes de cada actuación y el 14,3\% mostró de un alto índice de ansiedad durante la actuación. Teniendo en cuenta que la mayoría de los estudios hechos sobre la ansiedad, se han realizado en muy variados grupos de pacientes psiquiátricos, resulta muy interesante el hecho de que una muestra extraída de una población normal con un buen funcionamiento a nivel social, informe sobre tan devastadora ansiedad, en un campo que está relacionado con sus estudios.

Los síntomas que aparecen con mayor frecuencia son las palpitaciones y los temblores, ambos formando parte del grupo de síntomas fisiológicos. La sudoración apa- rece en cuarto lugar, con lo que parece ser que los síntomas fisiológicos son bastante importantes en estos casos. Normalmente estos síntomas se dan más entre los alumnos de música clásica que entre los de jazz. Sin embargo, también existían síntomas cognitivo-conductuales con relativa frecuencia. Por otro lado existían diferencias significativas entre hombres y mujeres con respecto a la ansiedad antes de la actuación y también respecto de la necesidad de ayuda. En cuanto a la reacción de alerta, sólo las palpitaciones resultaron estadísticamente significativas según el sexo de pertenencia aunque más alta en las mujeres que en los hombres. Los temblores, por otro lado, se hallaban entre los síntomas más frecuentes.

También llamaba la atención el hecho de que no hubiera diferencias de grupo con respecto al nivel educativo, pudiendo indicar ello que la AAM no desaparece con el paso del tiempo, como debiera esperarse tras los resultados de Wesner y col. (1990). Nuestros datos no incluyeron a profesionales, $\mathrm{y}$ por lo tanto no podemos sacar conclusiones 
acerca de si éstos exhiben un nivel de ansiedad diferente o no. También nuestros datos pueden estar apuntando otra posibilidad: que los estudiantes no tengan la suficiente experiencia como para superar la AAM. Si así fuera, podría ser interesante desde el punto de vista pedagógico trabajar sobre ello en un futuro.

Los hallazgos resultantes de este análisis en los diferentes grupos de instrumentos son bastante interesantes. Los síntomas como "Pánico" y "Pensamiento de que ésto no saldrá bien" fueron señalados por parte de los pianistas y los músicos de cuerda. Además existía un alto índice de ansiedad especialmente entre los músicos de teclado y los de cuerda, sugiriendo como cierta nuestra hipótesis de que son los músicos solistas los que con mayor frecuencia presentan problemas relacionados con la AAM frente a lo que sucede con los músicos de orquesta. La memoria, la concentración y las habilidades motoras exigidas a un pianista o a un músico de cuerda en una actuación, son importantes en cuanto a los resultados obtenidos. Entre los diferentes grupos de instrumentos se plantearon dos hipótesis: que se daban diferencias generales entre los diferentes grupos de instrumentos, y que existían diferencias específicas entre los solistas y la orquesta. La primera hipótesis no se vio respaldada por los presentes resultados, pero sí la segunda. Los músicos de piano y los de cuerda decían tener más AAM que los músicos de jazz, posiblemente debido al hecho de que los pianistas y los músicos de cuerda suelen realizar las actuaciones en solitario, mientras que los de jazz lo hacían en grupo.

La AAM se relacionó con distintas situaciones, por un lado con las actuaciones en solitario donde se obtuvieron resultados estadísticamente significativos, tanto antes como durante la actuación, lo cual apoyó la hipótesis de los solistas frente a la orquesta. Sin embargo ello fue más significativo antes de la interpretación que durante la misma, de ahí la importancia de la ansiedad expectante. Las respuestas: "Depende de la importancia que tenga el hacer todo bien" y "Depende de cómo me sienta de estresado" resultaron ser significativas estadísticamente. El perfeccionismo que busca conseguir una interpretación correcta de acuerdo con la tradición musical es muy importante en la música clásica occidental, siendo característica típica de ella.

Se deberían realizar estudios más profundos para establecer si esto es distinto en el caso de los músicos de jazz. "Si se puede improvisar, o no" resultó significativo sólo durante la actuación. Estas dos cuestiones pueden estar en relación con diferencias entre los estudiantes de música clásica y los de jazz. La facilidad para la improvisación puede no afectar a la ansiedad de antes de la actuación, pero sí ser de gran importancia durante la misma. Sin embargo, se debería realizar un estudio más profundo sobre las diferencias existentes entre los músicos de jazz y los improvisadores para contrastarlo con lo que sucede entre los músicos "clásicos". Sorprendentemente, la "preparación" no resultó significativa en este aspecto. Esto resulta difícil de interpretar. La preparación, se considera como el factor más importante para regular las variaciones de la ansiedad entre los músicos en general y en la tradición musical clásica en particular. Esto puede tener implicaciones pedagógicas. En la preparación de las actuaciones en el caso de la alta educación musical, se pone mucha energía. Se necesitan comprobaciones más profundas para establecer si pueden existir otros factores más importan- 
tes que la preparación, en relación con la AAM.

La AAM, tanto antes como durante la actuación, está relacionada positivamente con la NA. Así, nuestra hipótesis acerca de que los rasgos de ansiedad deben estar relacionados con la AAM, se ve confirmada por los resultados.

Las encuestas pueden resultar limitadas cuando recogen conclusiones sobre un grupo particular, ya que los estudiantes que responden al cuestionario pueden representar sólo a un subgrupo particular con un interés especial en los asuntos que se investigan, pudiendo por tanto, ofrecer los resultados parciales. Sin embargo, si se tiene en cuenta el alto porcentaje de respuestas obtenido en el presente estudio, es poco probable que todos estos estudiantes tuvieran un interés especial en este tema. Por ello, es razonable concluir que la ansiedad y la necesidad de ayuda, recogidas en este estudio, son algo real y no parcial.

Basándose en el presente artículo, se sugiere realizar investigaciones más profundas en los siguientes aspectos. Las comparaciones entre estudiantes de música clásica y estudiantes de jazz pueden ser interesantes para poder establecer si estos grupos de alumnos difieren realmente en relación a la AAM, y si es así, qué causas, en términos de motivación y de personalidad, pueden atribuirse a ello. Todavía más aún se deberían realizar investigaciones que pudieran ofrecer comparaciones entre profesionales y grupos de estudiantes para conseguir establecer cómo se desarrolla la AAM. Los estudios longitudinales podrían hacer posible la comparación de grupos en diferentes momentos, permitiéndonos descubrir los aspectos acerca de desarrollo de la AAM. Esto sería especialmente interesante en Noruega, dada la "frescura" que aún caracteriza a este país en cuanto a su cultura musical.

\section{Conclusiones}

Estos resultados revelan que la AAM y los síntomas relacionados con la AAM entre los estudiantes noruegos de música, no difieren de forma substancial de los estudios llevados a cabo en otros países europeos o en los Estados Unidos. Muestran también una necesidad de superar los problemas relacionados con la AAM, e informan sobre la necesidad de ayuda. Los principales hallazgos además de éste son: 1. Existen diferencias sexuales con respecto a la AAM, siendo las mujeres las que refieren mayor ansiedad (tanto fisiológica como cognitiva). Las mujeres sufren también más pensamientos intrusivos durante la actuación que los hombres. 2. La AAM no parece desaparecer con el tiempo. 3. Los pianistas y los músicos de cuerda informan sobre una mayor ansiedad con más frecuencia que entre los músicos de otro tipo de instrumentos. 4. Los estudiantes de jazz suelen tener una actitud diferente ante las actuaciones cuando se los compara con los estudiantes de música clásica, pero se necesitan investigaciones más profundas para poder llegar a nuevas conclusiones. 5 . Las actuaciones en solitario parecen provocar más ansiedad que las actuaciones en el conjunto de la orquesta. 6. La destreza en la improvisación está relacionada con la AAM durante las actuaciones, por lo que dicha habilidad no parece afectar a la ansiedad expectante. 7. Los resultados obtenidos tienen muchas implicaciones pedagógicas, específicamente las relacionadas con una educación musical de mayor nivel. 


\section{References}

ABEL, J.L.,LARKIN, K.T. Anticipation of performance among musicians: Physiological Arousal, Confidence, and State-Anxiety. Psychology of Music, 18,171-182,1990.

BARNEY DEWS, C.L., WILLIAMS, M.S. Student musicians' personality styles, Stresses, and Coping Patterns. Psychology of Music, 17, 37-47. 1989.

BRODSKY, W. Music Performance Anxiety Reconceptualized:A Critique of Cur rent Research Practices and Findings. Medical Problems of Performing Artists, 11, 88-98. 1996.

CLARK, D.B. Performance-related medical and psychological disorders in instrumental Musicians. Annals of Behavioural Medicine, 11, 28-34. 1939.

CLARK, D.B., AGRAS, W.S. The assessment and treatment of performance anxiety in musicians. American Jour nal of Psychiatry, 148, 598-605. 1991.

CRAIG, K. Physiological arousal as a function of imagined, vicarious and direct stress experiences. Journal of Abnormal Psychology, 73, 513-520. 1968.

CRASKE, M.G., CRAIG, D.G. Musical performance anxiety: The three-systems model and self-efficacy theory. Behaviour Research and Therapy, 22, 263-280. 1984.

DOCTOR, R.M., KAHN, A.P. The encyclopaedia of phobias, fears and anxieties. New York. 1989.

EYSENCK, H.J., EYSENCK, S.B.G., BARRETT, P.A revised version of the psychoticism scale. Personality and Individual Differences 6, 21-29. 1985.

FISHBEIN, M., MIDDLESTADT, S.E., OTTATI, V., STRAUS, S.,ELLIS, A. Medical problems among ICSOM musicians: Overview of a national survey. Medical Pro blems of Performing Artists, 3, 1-8. 1988.

HERRESTHAL,H. Med spark i gulvet og quinter i bas sen. Musilalske og politiste bilder fra nasjanalromanti klens gjennombrudd i Norge (Musical and political pictures from the break of the national romantics in Norway). Oslo. 1993.

HAVAS, K. Stage Fright:Its causes and cures. London: Bosworth. 1973.

JACKSON, J.M., LATANÉ. All alone in front of all those people: stage fright as function of number and type of co-performers and audience. Journal of Personality and Social Psychology, 40, 73-85. 1981.

KEMP, A.E. The Musical Temperament. Oxford University Press, 2000.
KIMIMÄKI, M. (1995). Test anxiety, below-capacity performance, and poor test performance: intrasubject approach with violin students. Personality of Individual Differences, 18, 47-55. 1995.

KUBZANSKY, L.D., STEWART, A.J. (1999). At The Intersection of Anxiety, Gender, and Performance. Journal of Social and Clinical Psychology, 18, 76-97. 1999.

LEHRER, P.M. A review of the approaches to the management of tension and stage fright in music performance, Journal of Research in Music Education, 35, 143-152. 1987.

LONIGAN, C.J., HOOE, E.S., DAVID, C.F., KISTNER, J.A. Positive and Negative Affectivity in Children: Confirmatory Factor Analysis of a Two-Factor Model and Its relation to Symptoms of Anxiety and Depression. Jour nal of Consulting and Clinical. Psychology, 67, 374-386. 1999.

MOR, S., DAY, H. I.,FLETT, G. L.,HEWITT, P.L. Perfectionism, control, and components of performance anxiety in professional artists. Cognitive Therapy and Research, 19, 207-225. 1995.

NORBERT, E. The civilising process. Oxford: Oxford University Press, 1994.

SALMON, P.G. A psychological perspective on musical performance anxiety: A review of the literature. Medical Problems of Performing Artists, 5(3), 2-11. 1990.

STEPTOE, A., FIDLER, H. Stage fright in orchestral musicians:A study of cognitive and behavioural strategies in performance anxiety. Brilish Journal of Psychology, 78, 241-49. 1987.

WATSON, D., CLARK, L.A., TELLEGEN, A. Development and validation of brief measures of positive and negative affect: The PANAS scales. Journal of Personality and Social Psychology, 54, 1063-1070. 1988.

WESNER, R.B.,NOYES, R. Jr. DAVIS, T.L. The occurrence of performance anxiety among musicians. Journal of Affective Disorders, 18, 177-185. 1990.

WILSON, G.D. In: The Social Psychology of Music. Hargreaves, D.J. and North,A.C. (eds.). Oxford University Press, 2000.

Dirección para correspondencia:

Marianne Kaspersen,

Departamento de Psiquiatría y Medicina Comportamental, Universidad de Ciencia y Tecnología,

P.O. Box 3008 Lade,

NO-7441 Trondheim

NORUEGA

E-mail: marianne.kaspersen@medisin.ntnu.no 


\section{Nota}

Un manuscrito preliminar del presente estudio fue presentado a la Sociedad para la Investigación en Psicología de la Música y la Educación Musical, en una Conferencia en Leicester en abril de 2000.

Esta investigación ha sido realizada bajo los auspicios de la Sociedad Noruega de Salud Mental y la Universidad Noruega de Ciencia y Tecnología en Trondheim. Queremos agradecer a la dirección, a la administración y a los estudiantes y profesores de la Academia Musical "Grieg" en Bergen y al

Conservatorio de Música en Trondheim NTHU, el haber participado en este proyecto. 